Virginia Commonwealth University VCU Scholars Compass

2003

\title{
Nitrogen-induced magnetic transition in small chromium clusters
}

\author{
Q.Wang
}

Virginia Commonwealth University

Q. Sun

Virginia Commonwealth University

B. K. Rao

Virginia Commonwealth University

P. Jena

Virginia Commonwealth University, pjena@vcu.edu

Y. Kawazoe

Tohoku University

Follow this and additional works at: http://scholarscompass.vcu.edu/phys_pubs

Part of the Physics Commons

Wang, Q. Sun, Q. Rao, B. K., et al. Nitrogen-induced magnetic transition in small chromium clusters. The Journal of Chemical Physics 119, 7124 (2003). Copyright (C) 2003 AIP Publishing LLC.

\section{Downloaded from}

http://scholarscompass.vcu.edu/phys_pubs/167

This Article is brought to you for free and open access by the Dept. of Physics at VCU Scholars Compass. It has been accepted for inclusion in Physics Publications by an authorized administrator of VCU Scholars Compass. For more information, please contact libcompass@vcu.edu. 


\title{
Nitrogen-induced magnetic transition in small chromium clusters
}

\author{
Q. Wang, Q. Sun, B. K. Rao, and P. Jena \\ Physics Department, Virginia Commonwealth University, Richmond, Virginia 23284-2000 \\ Y. Kawazoe \\ Institute for Material Research, Tohoku University, Sendai, 980-8577, Japan
}

(Received 18 March 2003; accepted 18 July 2003)

\begin{abstract}
Using density functional theory with generalized gradient approximation for exchange and correlation, we show that otherwise antiferromagnetically coupled chromium atoms in very small chromium clusters couple ferromagnetically when doped with a nitrogen atom, thus leading to giant magnetic moments. For example, the magnetic moment of $\mathrm{Cr}_{2} \mathrm{~N}$ is found to be $9 \mu_{\mathrm{B}}$ while that of $\mathrm{Cr}_{2}$ is $0 \mu_{\mathrm{B}}$. Strong bonding between $\mathrm{Cr}$ and $\mathrm{N}$ atoms brings about this magnetic transition. The $\mathrm{Cr}$ atoms nearest neighbor to $\mathrm{N}$ couple ferromagnetically with each other and antiferromagnetically with nitrogen. The significance of these results in understanding the ferromagnetic order in Cr-doped GaN is discussed. (C) 2003 American Institute of Physics. [DOI: 10.1063/1.1607958]
\end{abstract}

\section{INTRODUCTION}

Among the $3 d$ transition metals, $\mathrm{Cr}$ and Mn exhibit very contrasting behavior while sharing some common features. With a $3 d^{5} 4 s^{1}$ configuration, $\mathrm{Cr}$ atom binds strongly with another $\mathrm{Cr}$ atom and the resulting sextuple bonding in $\mathrm{Cr}_{2}$ dimer ${ }^{1}$ yields a very short bond $(1.68 \AA)$ and a large binding energy $(1.44 \mathrm{eV})$. On the other hand, a Mn atom, with a configuration of $3 d^{5} 4 s^{2}$, binds very weakly with another Mn atom. ${ }^{2}$ The bond length of the $\mathrm{Mn}_{2}$ dimer-namely, 3.5 $\AA$-is the largest among any $3 d$ transition-metal dimers and its binding energy is vanishingly small. Small clusters of Mn containing five atoms or fewer are ferromagnetic while clusters of $\mathrm{Cr}$ in the same size range are antiferromagnetic. In the bulk phase both $\mathrm{Mn}$ and $\mathrm{Cr}$ are antiferromagnetic. In this regard, it is interesting to note that ligated $\mathrm{Mn}_{4}$ clusters have shown ferromagnetic behavior and have been proposed as molecular magnets for quantum devices. ${ }^{3}$ Therefore, it should be interesting to examine the magnetic behavior of $\mathrm{Cr}$ clusters to see if they can also behave as molecular magnets under specific conditions.

Recently, Mn-doped GaN has been found to be ferromagnetic, although the controversy regarding the value of its Curie point still persists. ${ }^{4}$ It has been shown that nitrogenation of small Mn clusters not only enhances their binding energy substantially, but also the ferromagnetic coupling between $\mathrm{Mn}$ atoms leads to giant magnetic moments. ${ }^{5}$ For example, the total magnetic moment of $\mathrm{Mn}_{5} \mathrm{~N}$ is $22 \mu_{\mathrm{B}}$. Since Cr-doped $\mathrm{GaN}$ has just been discovered to be ferromagnetic, ${ }^{6}$ one wonders if this coupling is mediated by nitrogen and if clustering of $\mathrm{Cr}$ around nitrogen is energetically favorable.

To understand this, we have calculated the equilibrium geometries, electronic structure, total energies, and magnetic moments of $\mathrm{Cr}_{n} \mathrm{~N}(n \leqslant 5)$ clusters by using the density functional theory (DFT) and the generalized gradient approximation (GGA) to the exchange-correlation potential. We note that an earlier calculation ${ }^{7}$ had shown that small $\mathrm{Cr}$ clusters are antiferromagnetic and the total magnetic moments of $\mathrm{Cr}_{n}$ clusters are $0 \mu_{\mathrm{B}}, 6 \mu_{\mathrm{B}}, 0 \mu_{\mathrm{B}}$, and $4.65 \mu_{\mathrm{B}}$, for $n=2,3,4$, and 5, respectively. While we find significant differences between our calculated equilibrium geometries and magnetic properties of some of the $\mathrm{Cr}_{n}(n \leqslant 5)$ clusters with the previous calculation, these clusters are still antiferromagnetically coupled. On the other hand, the total magnetic moments of $\mathrm{Cr}_{n} \mathrm{~N}$ clusters are $9 \mu_{\mathrm{B}}, 13 \mu_{\mathrm{B}}, 9 \mu_{\mathrm{B}}$, and $3 \mu_{\mathrm{B}}$, for $n=2,3,4$, and 5 , respectively. In the following we discuss the origin of this magnetic transition and its implications for the understanding of ferromagnetism in Cr-doped GaN. In Sec. II we provide a brief outline of our theoretical procedure. The results are discussed in Sec. III and summarized in Sec. IV.

\section{THEORETICAL PROCEDURE}

The calculations are carried out using molecular orbital theory where the wave function of the cluster is represented by a linear combination of atomic orbitals centered at individual atomic sites. We describe the atomic orbitals by an all-electron Gaussian basis, 6-311G**, which is available in the GAUSSIAN 98 code. ${ }^{8}$ The total energy of the cluster is calculated using the DFT-GGA level of the theory. We have used the hybrid BPW91 form and the GAUSSIAN 98 code for our computations. Cr contains $d$ electrons and one does not know a priori the ground-state spin configuration of a given cluster. Therefore, we have performed calculations for all allowable spin multiplicities $M=2 S+1$, starting with a spinsinglet configuration for the even-electron system and spindoublet configuration for the odd-electron system. For a given spin multiplicity, we optimize the geometrical structure of a cluster by starting with different initial configurations and optimizing the geometry without symmetry constraints. The ground-state structure and preferred spin multiplicity are obtained from the minimum in the total energy. Except for the smallest clusters no frequency calculation was performed in order to avoid excessive computation. However, many random initial configurations were used to make reasonably sure that one may not end up with a local minimum. In the following we discuss our results. 
TABLE I. Binding energies/atom (eV), magnetic moments/atom $\left(\mu_{\mathrm{B}}\right)$, and symmetry of the ground state of the $\mathrm{Cr}_{n}(n \leqslant 5)$ clusters calculated in the present paper and compared with calculations of previous authors.

\begin{tabular}{cccccc}
\hline \hline Method & $n$ & $\begin{array}{c}\text { Present } \\
\text { DFT-GGA } \\
\text { all-electron } \\
\text { collinear spins }\end{array}$ & $\begin{array}{c}\text { Cheng-Wang } \\
\text { DFT-LSDA } \\
\text { frozen core } \\
\text { collinear spins }\end{array}$ & $\begin{array}{c}\text { Kohl-Bertsch } \\
\text { DFT-LSDA } \\
\text { pseudopotential } \\
\text { noncollinear spins }\end{array}$ & Expt. \\
\hline$E_{b}$ (eV/atom) & 2 & 0.97 & 1.14 & 0.99 & $0.72^{\mathrm{c}}$ \\
& 3 & 0.98 & 1.10 & & - \\
Magnetic moment & 4 & 1.34 & 1.46 & & - \\
$\left(\mu_{\mathrm{B}}\right.$ /atom) & 5 & 1.50 & 1.70 & 0.00 & - \\
& 3 & 0.00 & 0.00 & 0.69 & - \\
Ionization potential & 2 & 2.00 & 2.00 & 0.00 & - \\
$(\mathrm{eV})$ & 5 & 0.00 & 0.00 & & - \\
& 3 & 0.40 & 0.92 & & - \\
Structural & 4 & 4.07 & & & - \\
symmetry & 5 & 5.74 & & & - \\
& 2 & 5.21 & $D_{h}$ & & \\
& 3 & $C_{s}$ & $D_{h}$ & & - \\
\hline \hline
\end{tabular}

${ }^{a}$ Reference 7

${ }^{\mathrm{b}}$ Reference 9 .

\section{RESULTS AND DISCUSSIONS}

Calculations of the equilibrium geometries, electronic structures, magnetic moments, and binding energies were carried out for $\mathrm{Cr}_{n}$ and $\mathrm{Cr}_{n} \mathrm{~N}$ clusters for $n \leqslant 5$. Although the primary focus of this work is to examine the role of nitrogen doping on the stability and magnetic properties of Cr clusters, we first describe our results on pure $\mathrm{Cr}_{n}$ and compare with those available in the literature. ${ }^{7,9}$

\section{A. $\mathrm{Cr}_{n}(n \leqslant 5)$ clusters}

Cheng and Wang ${ }^{7}$ have studied the structure, binding energy, and magnetic properties of $\mathrm{Cr}_{n}(n \leqslant 15)$ clusters using density functional theory, local spin density approximation (LSDA), and numerical atomic bases with frozen $\mathrm{Cr}$ $1 s 2 s 2 p$ cores. According to these authors, an exhaustive structural search for cluster structures was performed by fully optimizing the geometries without imposing symmetry constraints starting from a wide variety of trial structures. Their main conclusions are that (1) $\mathrm{Cr}_{n}$ clusters exhibit a dimer-growth pattern until $n \leqslant 11$, beyond which the clusters begin to mimic a bcc growth pattern which is the crystal structure of bulk $\mathrm{Cr}$ and (2) the coupling between the $\mathrm{Cr}$ spins is antiferromagnetic and the total magnetic moment of $\mathrm{Cr}_{2}, \mathrm{Cr}_{3}, \mathrm{Cr}_{4}$, and $\mathrm{Cr}_{5}$ clusters are $0,6 \mu_{\mathrm{B}}, 0$, and $4.65 \mu_{\mathrm{B}}$, respectively. These authors have treated the spins to be collinear: i.e., they are either parallel or antiparallel. Recently, Kohl and Bertsch ${ }^{9}$ have studied small Cr clusters containing up to 13 atoms using pseudopotentials and by allowing the spins to assume a canted or noncollinear configuration within the framework of the LSDA. To facilitate comparison with our calculations, we summarize in Table I the results of these authors for clusters of up to five atoms.

The $\mathrm{Cr}$ dimer is one of the most studied systems ${ }^{1}$ in the transition-metal series. Both groups ${ }^{7,9}$ of authors find $\mathrm{Cr}_{2}$ to be antiferromagnetically coupled. The calculated bond length
${ }^{\mathrm{c}}$ Reference 13.

${ }^{\mathrm{d}}$ Reference 12 . and binding energy/atom of $1.69 \AA$ and $1.14 \mathrm{eV}$ by Cheng and $\mathrm{Wang}^{7}$ and $1.72 \AA$ and $0.99 \mathrm{eV}$ by Kohl and Bertsch ${ }^{9}$ compare favorably with the experimental value ${ }^{1}$ of $1.68 \AA$ and $0.72 \mathrm{eV} /$ atom, respectively. Note that the difference in the calculated binding energies of $0.15 \mathrm{eV} / \mathrm{atom}$ by these two groups can only be attributed to different numerical procedures as both authors use the local spin density approximation and frozen core or pseudopotential. For $\mathrm{Cr}_{3}$ Cheng and Wang ${ }^{7}$ find the structure to have $C_{2 v}$ symmetry in which two $\mathrm{Cr}$ atoms remain dimer like while the third atom sits at an apex position of the isosceles triangle. The coupling between the nearest-neighbor atoms is antiferromagnetic and the apex atom is responsible for the entire $6 \mu_{\mathrm{B}}$ magnetic moment. Kohl and Bertsch, ${ }^{9}$ on the other hand, have pointed out that $\mathrm{Cr}_{3}$ is a classic case of a frustrated system where the spin of the apex atom does not know whether to point up or down if the spins are constrained to be collinear. However, once the spins are allowed to be noncollinear, the frustration is removed and the energy can be lowered. Indeed, they find the total moment of $\mathrm{Cr}_{3}$ to be $2 \mu_{\mathrm{B}}$ and the noncollinear configuration lies $0.083 \mathrm{eV} /$ atom lower in energy than the collinear state. Note that the choice of basis sets (i.e., all-electron versus pseudopotential or frozen core potential), choice of exchange correlation functional, and other numerical details, as discussed in the above, can lead to an inaccuracy in the total binding energy of a cluster by about $0.2 \mathrm{eV}$ or larger. $\mathrm{Cr}_{4}$ has been found to have a collinear ground state with the lowest noncollinear state lying $0.12 \mathrm{eV} /$ atom higher in energy. On the other hand, the $\mathrm{Cr}_{5}$ ground state is noncollinear with the collinear state lying $0.054 \mathrm{eV} /$ atom higher in energy. The magnetic moments/atom calculated by Kohl and Bertsch ${ }^{9}$ are smaller than those if the states are collinear with the exception of $\mathrm{Cr}_{4}$ where the noncollinear configuration has a bigger total magnetization than the collinear configuration. This is not surprising as the total magnetization of the collinear con- 


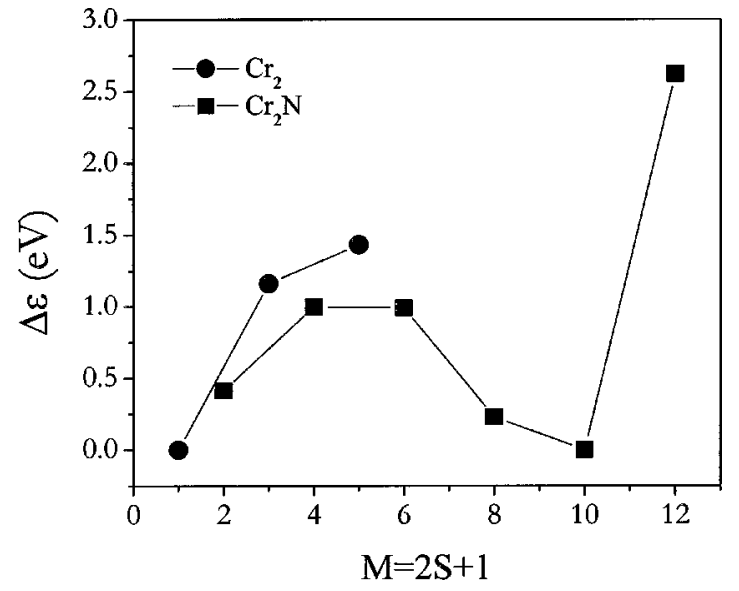

FIG. 1. Relative energies $\Delta \varepsilon$ of various spin multiplicities measured with respect to the ground state. The solid circle and solid square refer, respectively, to $\mathrm{Cr}_{2}$ and $\mathrm{Cr}_{2} \mathrm{~N}$.

figuration of $\mathrm{Cr}_{4}$ is zero. Unfortunately, there are no experiments, except that for $\mathrm{Cr}_{2}$, with which these results have been compared.

In our calculation we have used an all-electron basis. The exchange correlation has been treated within the GGA using the hybrid BPW91 functional. ${ }^{8}$ However, we have used the collinear configuration as the inclusion of vector spins within the GGA is still under study for bulk materials ${ }^{10}$ and no theory is available for this for clusters where the lack of symmetry does not allow the use of packages like the VASP code. ${ }^{11}$ For a given cluster we have optimized the structure for all possible spin multiplicities starting with singlets for even- and doubles for odd-electron systems. In Figs. 1-4, we plot the energies calculated with respect to the ground-state spin configuration for $\mathrm{Cr}_{2}, \mathrm{Cr}_{3}, \mathrm{Cr}_{4}$, and $\mathrm{Cr}_{5}$ clusters. Note that these energy differences are not monotonic. While the energy difference between two successive spin multiplicities may be small in some cases, they can be as much as $1 \mathrm{eV}$ in some other cases.

In Table I we list our results corresponding to the ground-state spin configuration. In the following we will compare these results with the above calculations and avail-

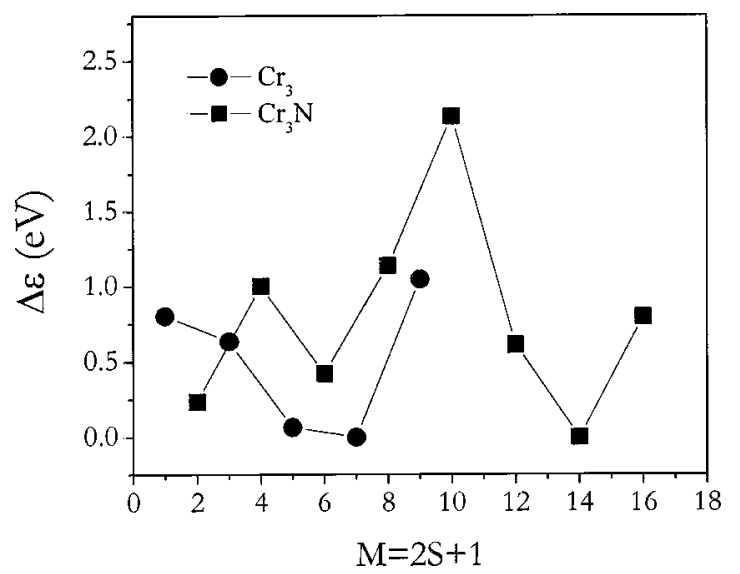

FIG. 2. Relative energies $\Delta \varepsilon$ of various spin multiplicities measured with respect to the ground state. The solid circle and solid square refer, respectively, to $\mathrm{Cr}_{3}$ and $\mathrm{Cr}_{3} \mathrm{~N}$.

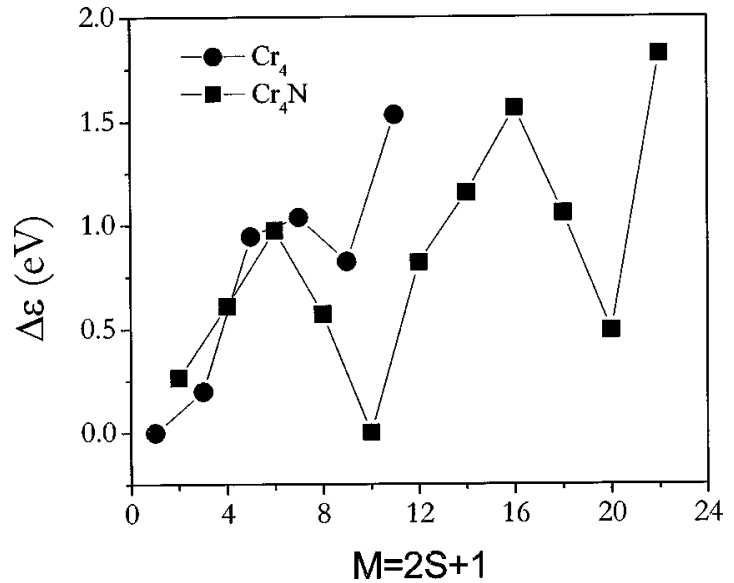

FIG. 3. Relative energies $\Delta \varepsilon$ of various spin multiplicities measured with respect to the ground state. The solid circle and solid square refer, respectively, to $\mathrm{Cr}_{4}$ and $\mathrm{Cr}_{4} \mathrm{~N}$.

able experiments. We begin by giving the equilibrium geometries of $\mathrm{Cr}_{n}(n \leqslant 5)$ clusters along with their higher-energy isomers in Figs. 5-7. In agreement with previous authors ${ }^{7,9}$ we find $\mathrm{Cr}_{2}$ to be antiferromagnetic with a binding energy of $0.97 \mathrm{eV} /$ atom and a bond length of $1.66 \AA$. These results agree well with the experimental values of $0.72 \mathrm{eV} /$ atom and $1.68 \AA$.

We have identified three isomers of $\mathrm{Cr}_{3}$ [see Figs. 5(b)5(d)]. The ground-state geometry of $\mathrm{Cr}_{3}$ [Fig. 5(b)] is found to have a $C_{s}$ symmetry with two $\mathrm{Cr}$ atoms lying at a distance of $1.71 \AA$ (dimer like) while the third atom lies 2.91 and 2.39 $\AA$ from each of the other two $\mathrm{Cr}$ atoms. Cheng and Wang, ${ }^{7}$ on the other hand, found the ground state of $\mathrm{Cr}_{3}$ to have a $C_{2 v}$ symmetry. We find the $C_{2 v}$ structure to lie $0.24 \mathrm{eV}$ above the ground state. However, a third isomer in the form of a linear chain [Fig. 5(d)] is nearly degenerate with the ground-state structure as its energy is only $0.034 \mathrm{eV}$ higher than the most stable structure. Note that the spin frustration noted by Kohl and Bertsch ${ }^{9}$ disappears in the $C_{s}$ structure [Fig. 5(b)] as well as in the linear structure [Fig. 5(d)]. In Fig. 5(b), the apex atom is asymmetrical and thus views the other two atoms differently. It couples ferromagnetically

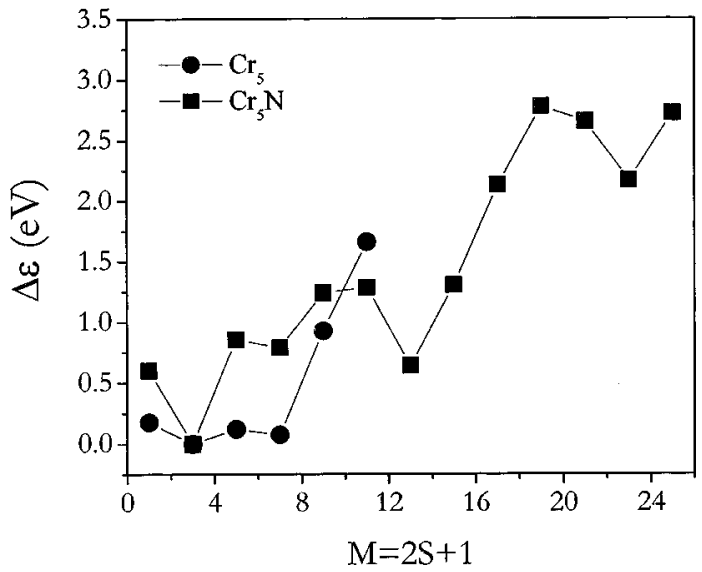

FIG. 4. Relative energies $\Delta \varepsilon$ of various spin multiplicities measured with respect to the ground state. The solid circle and solid square refer, respectively, to $\mathrm{Cr}_{5}$ and $\mathrm{Cr}_{5} \mathrm{~N}$. 


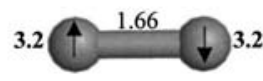

$\mu_{\text {total }}=0 \mu_{\mathrm{B}}, \mathrm{E}_{\mathrm{b}}=0.97 \mathrm{eV} /$ atom $\mathrm{IP}=6.07 \mathrm{eV}$ (a)

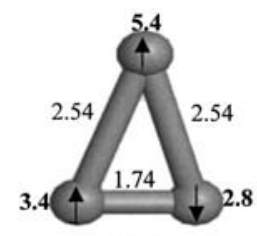

$\left(\mathrm{C}_{2 \mathrm{v}}\right)$

$\mu_{\text {total }}=6 \mu_{\mathrm{B}}, \Delta \varepsilon=0.241 \mathrm{eV}$ $\mathrm{IP}=4.66 \mathrm{eV}(\mathrm{c})$

FIG. 5. Geometries of the ground state and low-lying isomers of $\mathrm{Cr}_{2}$ and $\mathrm{Cr}_{3}$ clusters. Interatomic distance $(\AA)$, total magnetic moment $\left(\mu_{\mathrm{B}}\right)$, ionization potential IP $(\mathrm{eV})$, and relative energies $\Delta \varepsilon(\mathrm{eV})$ with respect to the ground state of each cluster are also given.

with the atom at a distance of $2.91 \AA$ and antiferromagnetically with the one at $2.39 \AA$. This is consistent with the result of Kohl and Bertsch who found the ground state of $\mathrm{Cr}_{2}$ to be antiferromagnetic at a distance of $1.72 \AA$ and ferromagnetic at a distance of $2.75 \AA$. Thus Fig. 5(b) lowers its energy by removing the frustration, not by having its spins canted, but by having its structure distorted. Note that the difference between the energy of Figs. 5(b) and 5(c) is 0.08 eV/atom, which is same as that gained by having noncollinear spins. Similarly, in Fig. 5(d), two atoms are dimer like. The third atom couples antiferromagnetically to the atom lying at a distance of $2.64 \AA$ and ferromagnetically to the one at a distance of $4.29 \AA$. Again, frustration is removed and the energy is lowered. All of these isomers have a total magnetic moment of $6 \mu_{\mathrm{B}}$ and two of the $\mathrm{Cr}$ atoms remain in a dimerlike configuration. The third atom is responsible for the majority of the magnetic moment of the $\mathrm{Cr}_{3}$ cluster. This, however, does not rule out the possibility that further energy lowering can still occur by allowing noncollinear spins on top of structural distortion.

We have identified four different isomers of $\mathrm{Cr}_{4}$. Their geometries, interatomic bond distances, magnetic moments, ionization potentials, binding energy of the ground state, and relative energies, calculated with respect to the ground-state structure, are given in Figs. 6(a)-6(d). The ground state of $\mathrm{Cr}_{4}$ [Fig. 6(a)] has a $\mathrm{D}_{2}$ symmetry where two $\mathrm{Cr}_{2}$-like dimers combine to form a twisted structure. A nearly degenerate structure in the form of a planar rhombus [Fig. 6(c)] shows no dimerlike growth. The other two high-energy isomers, which are also energetically degenerate, are shown in Figs. 6(b) and 6(d). Note that while one of them [Fig. 6(b)] shows a dimerlike growth, the other does not. Thus, unlike the observation of Cheng and Wang, ${ }^{7}$ we see the disappearance of dimerlike growth in clusters as small as $\mathrm{Cr}_{4}$. Note that the spin coupling is antiferromagnetic in all four isomers and the total magnetic moment is $0 \mu_{\mathrm{B}}$ in each case. Kohl

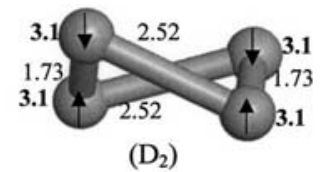

$\mu_{\text {total }}=0 \mu_{\mathrm{B}}, \mathrm{E}_{\mathrm{b}}=1.34 \mathrm{eV} /$ atom $\mathrm{IP}=5.75 \mathrm{eV}$ (a)

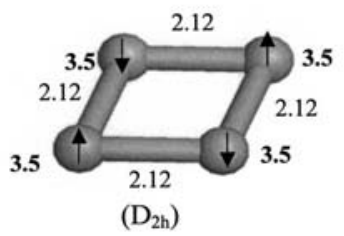

$\mu_{\text {total }}=0 \mu_{\mathrm{B}}, \Delta \varepsilon=0.01 \mathrm{eV}$ $\mathrm{IP}=4.94 \mathrm{eV}$ (c)

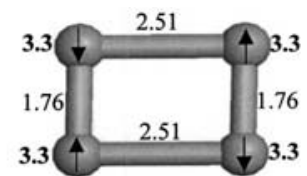

$\left(\mathrm{D}_{2 \mathrm{~h}}\right)$

$\mu_{\text {total }}=0 \mu_{\mathrm{B}}, \Delta \varepsilon=0.37 \mathrm{eV}$ $\mathrm{IP}=5.51 \mathrm{eV}$ (b)

FIG. 6. Geometries of the ground state (a) and low-lying isomers (b), (c), (d) of $\mathrm{Cr}_{4} \mathrm{~N}$ clusters. Interatomic distance $(\AA)$, total magnetic moment $\left(\mu_{\mathrm{B}}\right)$, ionization potential IP $(\mathrm{eV})$, and relative energies $\Delta \varepsilon(\mathrm{eV})$ with respect to the ground state of each cluster are also given.

and Bertsch $^{9}$ have found the ground state of $\mathrm{Cr}_{4}$ to have collinear spins with total magnetic moment of $0 \mu_{\mathrm{B}}$.

The equilibrium geometries, bond distances, magnetic moments, ionization potentials, and relative energies, calculated with respect to the ground state, of $\mathrm{Cr}_{5}$ cluster isomers are given in Figs. 7(a)-7(d). The ground-state structure [Fig. 7 (a)] and its nearly degenerate isomer [Fig. 7(b)] again show no sign of dimer growth. The higher-energy isomer lying $0.12 \mathrm{eV}$ above the ground state has a $C_{s}$ symmetry and also does not exhibit any dimer growth. The only isomer that

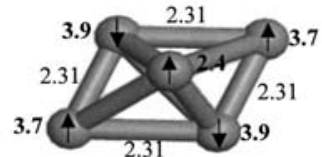

$\left(\mathrm{C}_{2 \mathrm{v}}\right)$

$\mu_{\text {total }}=2 \mu_{\mathrm{B}}, \mathrm{E}_{\mathrm{b}}=1.50 \mathrm{eV} /$ atom $\mathrm{IP}=5.21 \mathrm{eV}$ (a)

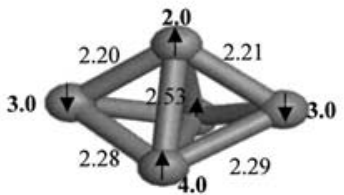

$\left(\mathrm{C}_{\mathrm{s}}\right)$

$\mu_{\text {total }}=4 \mu_{\mathrm{B}}, \Delta \varepsilon=0.12 \mathrm{eV}$ $\mathrm{IP}=4.99 \mathrm{eV}$ (c)

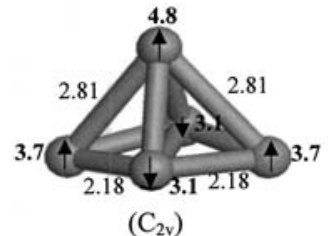

$\mu_{\text {total }}=6 \mu_{\mathrm{B}}, \Delta \varepsilon=0.06 \mathrm{eV}$ $\mathrm{IP}=4.98 \mathrm{eV}$ (b)

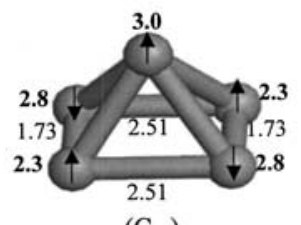

$\left(\mathrm{C}_{2 \mathrm{v}}\right)$

$\mu_{\text {total }}=2 \mu_{\mathrm{B}}, \Delta \varepsilon=1.01 \mathrm{eV}$ $\mathrm{IP}=4.87 \mathrm{eV}$ (d)
FIG. 7. Geometries of the ground state (a) and low-lying isomers (b), (c), (d) of $\mathrm{Cr}_{5} \mathrm{~N}$ clusters. Interatomic distance $(\AA)$, total magnetic moment $\left(\mu_{\mathrm{B}}\right)$, ionization potential IP $(\mathrm{eV})$, and relative energies $\Delta \varepsilon(\mathrm{eV})$ with respect to the ground state of each cluster are also given. 
shows dimer growth is given in Fig. 7(d), but it lies $1.01 \mathrm{eV}$ above the ground state. These results are different from those obtained by Cheng and Wang. We also find the total magnetic moment of the ground-state structure [Fig. 7(a)] as well as that of Fig. $7(\mathrm{~d})$ to be $2 \mu_{\mathrm{B}}$ in contrast to the $4.65 \mu_{\mathrm{B}}$ quoted by Cheng and Wang. We should recall that our calculated magnetic moments are obtained by optimizing the clusters for different allowable spin multiplicities and finding the value for which energy is minimum. It appears that Cheng and Wang may have used the aufbau principle to calculate the magnetic moments where one populates the single-particle energy levels of spin-up and -down states in increasing order. In systems such as Cr clusters, where the energy levels for spin up and down are close, the choice of the aufbau principle may lead to erroneous results. Kohl and Bertsch ${ }^{9}$ have found the $\mathrm{Cr}_{5}$ ground state to have noncollinear spins and hence a very different structure from those shown in Fig. 7. The structure with collinear spins lies 0.054 eV/atom above the noncollinear ground state.

As mentioned before, the energy differences between low-lying isomers as well as that between collinear and noncollinear spin configurations are rather small and are often within the accuracy of the numerical procedure. Thus it is very important to compare theoretical results with experiment to establish their accuracy. Unfortunately, no magnetic measurements are available to compare with the calculated moments in this size range. We have, therefore, calculated the vertical ionization potential-i.e., the energy necessary to remove an electron from a neutral cluster without changing its geometry. Note that the ensuing positively charged cluster can have a spin multiplicity that can differ from the neutral by \pm 1 . So we have calculated both these energies for all the isomers given in Figs. 5-7. The lower of these two energies is listed in the figures. In Table I we compare the vertical ionization potential calculated for the ground-state structure with available experiment. ${ }^{12}$ The agreement is very good and provides confidence in our calculated ground-state structures. The vertical ionization potentials of higher-energy isomers are given in Figs. 5-7. In particular, note that for $\mathrm{Cr}_{5}$ the isomer in Fig. 7(d) yields an ionization potential that is in maximum disagreement with experiment. The above analyses clearly point out the need for a thorough search for structural isomers and various spin multiplicities before identifying the ground-state structure and hence the growth mode.

To understand the electronic structure of these clusters and the contribution to the total magnetic moment of the clusters originating from $4 s$ and $3 d$ electrons of $\mathrm{Cr}$, we have calculated the electron occupation of $4 s$ and $3 d$ states of each atom for spin-up and -down configurations. The results are given in Tables II-V for $\mathrm{Cr}_{2}, \mathrm{Cr}_{3}, \mathrm{Cr}_{4}$, and $\mathrm{Cr}_{5}$ clusters. These will be compared with corresponding $\mathrm{N}$-doped clusters in the next section. Two points are to be noted: (1) The overlap between $s$ and $d$ states is rather small in these clusters as the occupancy of $4 s$ and $3 d$ states remains close to their free atom values of 1 and 5. (2) The magnetic moments arise from the spin polarization of both $s$ and $d$ electrons, although the contribution of $3 d$ electrons is more than five times larger than that from the $4 s$ electrons.
TABLE II. Valence electronic configuration with spin polarization for $\mathrm{Cr}_{2}$ and $\mathrm{Cr}_{2} \mathrm{~N}$ clusters.

\begin{tabular}{clcccccc}
\hline \hline \multirow{2}{*}{ Position } & & \multicolumn{2}{c}{$\mathrm{Cr}_{2}$} & & \multicolumn{2}{c}{$\mathrm{Cr}_{2} \mathrm{~N}$} \\
\cline { 3 - 4 } \cline { 6 - 7 } \cline { 6 - 7 } & & Spin & $4 s$ & $3 d$ & & $4 s$ & $3 d$ \\
\hline \multirow{2}{*}{$\mathrm{Cr} 1$} & spin up & 0.73 & 3.77 & & 0.87 & 4.29 \\
& spin down & 0.28 & 1.23 & & 0.04 & 0.37 \\
& total & 1.01 & 5.01 & & 0.91 & 4.60 \\
$\mathrm{Cr} 2$ & spin up & 0.28 & 1.23 & & 0.87 & 4.29 \\
& spin down & 0.73 & 3.77 & & 0.04 & 0.37 \\
& total & 1.01 & 5.01 & & 0.91 & 4.60 \\
$\mathrm{~N}$ & & & & & $2 s$ & $2 p$ \\
& spin up & & & & 0.97 & 1.71 \\
& spin down & & & & 0.91 & 2.38 \\
& total & & & & 1.88 & 4.09 \\
\hline \hline
\end{tabular}

\section{B. $\mathrm{Cr}_{n} \mathrm{~N}(n \leqslant 5)$ clusters}

In Fig. 8 we provide the geometries of the ground state and some higher-energy isomers of $\mathrm{Cr}_{n} \mathrm{~N}$ clusters. For $\mathrm{Cr}_{4} \mathrm{~N}$ we have found two isomers [Figs. 8(d) and 8(e)] while for $\mathrm{Cr}_{5} \mathrm{~N}$ we have identified three isomers [Figs. 8(f)-8(h)]. Note that the addition of $\mathrm{N}$ has a strong influence on the geometry of the $\mathrm{Cr}$ clusters as can be seen by comparing the results in Fig. 8 with those in Figs. 5-7. These result from a strong bonding between $\mathrm{Cr}$ and $\mathrm{N}$ atoms and will be discussed later in this paper. The $\mathrm{CrN}$ distance is $1.54 \AA$, which is enlarged as the $\mathrm{Cr}$ concentration increases. The $\mathrm{Cr}-\mathrm{N}-\mathrm{Cr}$ bond angle in $\mathrm{Cr}_{2} \mathrm{~N}$ is close to $120^{\circ}$ and this is maintained in $\mathrm{Cr}_{3} \mathrm{~N}$. In the ground-state structure of $\mathrm{Cr}_{4} \mathrm{~N}$ [Fig. 8(d)], the nitrogen atom is bonded to three $\mathrm{Cr}$ atoms, in keeping with the trivalent nature of $\mathrm{N}$. The structure where $\mathrm{N}$ occupies a tetrahedral position [Fig. 8(e)] is about $0.5 \mathrm{eV}$ above the ground state. Note that the ground state of $\mathrm{Cr}_{4}$ has a $\mathrm{D}_{2}$ structure where two $\mathrm{Cr}_{2}$-like dimers are twisted against each other, but in $\mathrm{Cr}_{4} \mathrm{~N}$, the four $\mathrm{Cr}$ atoms occupy a tetrahedral configuration and there is no signature of dimerlike growth. The structure of $\mathrm{Cr}_{5} \mathrm{~N}$ is again severely distorted from that of $\mathrm{Cr}_{5}$. Here the five $\mathrm{Cr}$ atoms occupy a trigonal bipyramid structure with the $\mathrm{N}$ atom capping one of the triangular faces.

TABLE III. Valence electronic configuration with spin polarization for $\mathrm{Cr}_{3}$ and $\mathrm{Cr}_{3} \mathrm{~N}$ clusters.

\begin{tabular}{clcccccc}
\hline \hline \multirow{2}{*}{ Position } & & \multicolumn{2}{c}{$\mathrm{Cr}_{3}$} & & \multicolumn{2}{c}{$\mathrm{Cr}_{3} \mathrm{~N}$} \\
\cline { 3 - 4 } \cline { 6 - 7 } \cline { 6 - 7 } & & Spin & $4 s$ & $3 d$ & & $4 s$ & $3 d$ \\
\hline \multirow{2}{*}{ rr1 } & spin up & 0.79 & 3.85 & & 0.79 & 4.28 \\
& spin down & 0.23 & 1.08 & & 0.32 & 0.23 \\
& total & 1.03 & 4.94 & & 1.11 & 4.51 \\
$\mathrm{Cr} 2$ & spin up & 0.39 & 1.31 & & 0.79 & 4.28 \\
& spin down & 0.63 & 3.75 & & 0.32 & 0.23 \\
& total & 1.02 & 5.06 & & 1.11 & 4.51 \\
$\mathrm{Cr} 3$ & spin up & 0.94 & 4.69 & & 0.79 & 4.28 \\
& spin down & 0.2 & 0.11 & & 0.32 & 0.23 \\
& total & 1.13 & 4.80 & & 1.11 & 4.51 \\
$\mathrm{~N}$ & & & & & $2 s$ & $2 p$ \\
& spin up & & & & 0.78 & 1.93 \\
& spin down & & & 0.91 & 2.45 \\
& total & & & 1.78 & 4.38 \\
\hline \hline
\end{tabular}


TABLE IV. Valence electronic configuration with spin polarization for $\mathrm{Cr}_{4}$ and $\mathrm{Cr}_{4} \mathrm{~N}$ clusters.

\begin{tabular}{clccccc}
\hline \hline \multirow{2}{*}{ Position } & & \multicolumn{2}{c}{$\mathrm{Cr}_{4}$} & & \multicolumn{2}{c}{$\mathrm{Cr}_{4} \mathrm{~N}$} \\
\cline { 3 - 4 } \cline { 6 - 7 } \cline { 5 - 7 } $\mathrm{n}$ Cr1 & Spin & $4 s$ & $3 d$ & & $4 s$ & $3 d$ \\
& spin up & 0.30 & 1.19 & & 0.74 & 4.29 \\
& spin down & 0.79 & 3.71 & & 0.15 & 0.39 \\
& total & 1.09 & 4.90 & & 0.89 & 4.68 \\
$\mathrm{Cr} 2$ & spin up & 0.79 & 3.71 & & 0.74 & 4.29 \\
& spin down & 0.30 & 1.19 & & 0.15 & 0.39 \\
& total & 1.09 & 4.90 & & 0.89 & 4.68 \\
$\mathrm{Cr} 3$ & spin up & 0.79 & 3.71 & & 0.58 & 0.47 \\
& spin down & 0.30 & 1.19 & & 0.69 & 4.37 \\
& total & 1.09 & 4.90 & & 1.26 & 4.83 \\
$\mathrm{Cr} 4$ & spin up & 0.30 & 1.19 & & 0.74 & 4.29 \\
& spin down & 0.79 & 3.71 & & 0.15 & 0.39 \\
& total & 1.09 & 4.90 & & 0.89 & 4.68 \\
$\mathrm{~N}$ & & & & & $2 s$ & $2 p$ \\
& spin up & & & 0.92 & 1.86 \\
& spin down & & & 0.91 & 2.39 \\
& total & & & 1.83 & 4.25 \\
\hline \hline
\end{tabular}

Two other isomers [Figs. 8(g) and $8(\mathrm{~h})$ ] were identified, but their energies were in excess of $0.5 \mathrm{eV}$ above the groundstate structure.

In Table VI we compare the binding energy $\Delta E$ of the $\mathrm{N}$ atom in $\mathrm{Cr}_{n} \mathrm{~N}$ clusters, calculated with respect to dissociation into $\mathrm{Cr}_{n}$ and $\mathrm{N}$, with that of the binding energy per atom, $E_{b}$ of the $\mathrm{Cr}_{n}$ cluster. We define these energies as

$$
\begin{aligned}
& \Delta E=-\left[E\left(\mathrm{Cr}_{n} \mathrm{~N}\right)-E\left(\mathrm{Cr}_{n}\right)-E(\mathrm{~N})\right], \\
& E_{b}=-\left[E\left(\mathrm{Cr}_{n}\right)-n E(\mathrm{Cr})\right] / n .
\end{aligned}
$$

We note that $\Delta E$ is substantially larger than $E_{b}$. Thus clustering of $\mathrm{Cr}$ around $\mathrm{N}$ is energetically favorable.

We now discuss the effect of $\mathrm{N}$ doping on the magnetic properties of $\mathrm{Cr}_{n}$ clusters. Once again, we have assumed a

TABLE V. Valence electronic configuration with spin polarization for $\mathrm{Cr}_{5}$

\begin{tabular}{|c|c|c|c|c|c|}
\hline \multirow[b]{2}{*}{ Position } & \multirow[b]{2}{*}{ Spin } & \multicolumn{2}{|c|}{$\mathrm{Cr}_{5}$} & \multicolumn{2}{|c|}{$\mathrm{Cr}_{5} \mathrm{~N}$} \\
\hline & & $4 s$ & $3 d$ & $4 s$ & $3 d$ \\
\hline \multirow[t]{3}{*}{$\mathrm{Cr} 1$} & spin up & 0.27 & 0.79 & 0.63 & 4.03 \\
\hline & spin down & 0.80 & 4.07 & 0.21 & 0.77 \\
\hline & total & 1.08 & 4.86 & 0.84 & 4.81 \\
\hline \multirow[t]{3}{*}{$\mathrm{Cr} 2$} & spin up & 0.65 & 4.18 & 0.63 & 4.03 \\
\hline & spin down & 0.64 & 0.51 & 0.21 & 0.77 \\
\hline & total & 1.29 & 4.69 & 0.84 & 4.81 \\
\hline \multirow[t]{3}{*}{$\mathrm{Cr} 3$} & spin up & 0.27 & 0.75 & 0.58 & 4.17 \\
\hline & spin down & 0.80 & 4.11 & 0.25 & 0.55 \\
\hline & total & 1.07 & 4.85 & 0.84 & 4.72 \\
\hline \multirow[t]{3}{*}{$\mathrm{Cr} 4$} & spin up & 0.65 & 4.18 & 0.52 & 0.54 \\
\hline & spin down & 0.64 & 0.51 & 0.69 & 4.28 \\
\hline & total & 1.29 & 4.69 & 1.12 & 4.81 \\
\hline \multirow[t]{3}{*}{$\mathrm{Cr} 5$} & spin up & 0.47 & 3.73 & 0.47 & 0.56 \\
\hline & spin down & 0.71 & 1.10 & 0.79 & 4.20 \\
\hline & total & 1.18 & 4.84 & 1.26 & 4.76 \\
\hline \multirow[t]{4}{*}{$\mathrm{N}$} & & & & $2 s$ & $2 p$ \\
\hline & spin up & & & 0.91 & 1.85 \\
\hline & spin down & & & 0.90 & 2.32 \\
\hline & total & & & 1.82 & 4.17 \\
\hline
\end{tabular}
and $\mathrm{Cr}_{5} \mathrm{~N}$ clusters.

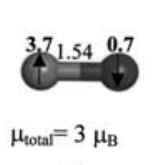

(a)

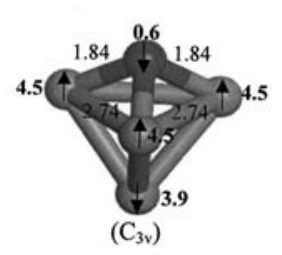

$\mu_{\text {total }}=9 \mu_{\mathrm{B}}, \Delta \varepsilon=0 \mathrm{eV}$

(d)

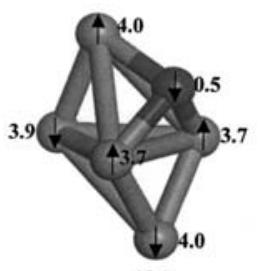

$\left(\mathrm{C}_{\mathrm{s}}\right)$

$\mu_{\text {ototal }}=3 \mu_{\mathrm{B}}, \Delta \varepsilon=0 \mathrm{eV}$

(f)

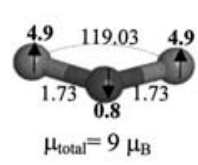

(b)

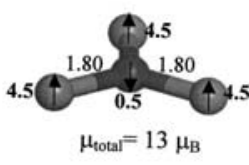

(c)

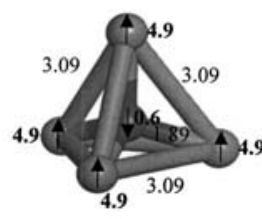

( Td)

$\mu_{\text {total }}=19 \mu_{\mathrm{B}}, \Delta \varepsilon=0.49 \mathrm{eV}$

(e)

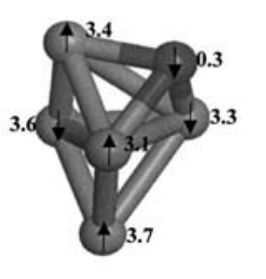

$\left(\mathrm{C}_{1}\right)$

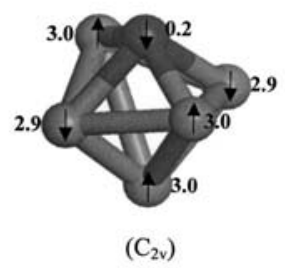

$\mu_{\text {total }}=3 \mu_{\mathrm{B}}, \Delta \varepsilon=0.85 \mathrm{eV}$

(h)

FIG. 8. Geometries of the ground state and low-lying isomers of $\mathrm{Cr}_{n} \mathrm{~N}$ ( $n$ $\leqslant 5)$ clusters. Interatomic distance $(\AA)$, total magnetic moment $\left(\mu_{\mathrm{B}}\right)$, ionization potential IP $(\mathrm{eV})$, and relative energies $\Delta \varepsilon(\mathrm{eV})$ with respect to the ground state of each cluster are also given. The dark atom corresponds to $\mathrm{N}$.

collinear spin configuration. Unlike the case of pure Cr clusters where frustration was removed by having noncollinear spins, there is no frustration in $\mathrm{Cr}_{n} \mathrm{~N}$ clusters. The presence of $\mathrm{N}$ breaks the symmetry and the $\mathrm{Cr}$ atoms are no longer equivalent. In Table VI we list the total magnetic moment of $\mathrm{Cr}_{n} \mathrm{~N}$ clusters and compare these with those of $\mathrm{Cr}_{n}$. Recall that $\mathrm{Cr}_{2}$ is antiferromagnetic with a total magnetic moment of $0 \mu_{\mathrm{B}}$. However, the coupling between the magnetic moments at the $\mathrm{Cr}$ site in $\mathrm{Cr}_{2} \mathrm{~N}$ is ferromagnetic. The magnetic moment at each of the $\mathrm{Cr}$ site is $4.9 \mu_{\mathrm{B}}$ and it couples antiferromagnetically with that of $\mathrm{N}$ which carries a small magnetic moment-namely, $0.8 \mu_{\mathrm{B}}$. The total magnetic moment of $\mathrm{Cr}_{2} \mathrm{~N}$ is $9 \mu_{\mathrm{B}}$-a substantial enhancement over that in $\mathrm{Cr}_{2}$. We see a similar trend in $\mathrm{Cr}_{3} \mathrm{~N}$. Here all $\mathrm{Cr}$ sites are ferromagnetically coupled and in turn each $\mathrm{Cr}$ moment is antiferromagnetically coupled to that of $\mathrm{N}$, which carries a small moment of $0.5 \mu_{\mathrm{B}}$. The total magnetic moment of $\mathrm{Cr}_{3} \mathrm{~N}$ is $13 \mu_{\mathrm{B}}$ while that of $\mathrm{Cr}_{3}$ is only $6 \mu_{\mathrm{B}}$. In $\mathrm{Cr}_{4} \mathrm{~N}$, the three $\mathrm{Cr}$ atoms bonding with the $\mathrm{N}$ atom are again coupled ferromagnetically while the fourth $\mathrm{Cr}$ atom having no bond with $\mathrm{N}$ couples antiferromagnetically with the other three $\mathrm{Cr}$ atoms. Thus it is because of cancellation between up and down spins that the total magnetic moment of $\mathrm{Cr}_{4} \mathrm{~N}$ is $9 \mu_{\mathrm{B}}$. Note that the antiferromagnetic coupling in $\mathrm{Cr}_{4}$ results in zero magnetic moment for the bare cluster. $\mathrm{In} \mathrm{Cr}_{5} \mathrm{~N}$, while the three $\mathrm{Cr}$ atoms bonded to $\mathrm{N}$ again couple ferromagnetically, the other two $\mathrm{Cr}$ atoms are antiferromagnetically coupled. The cancellation between up and down spins is, therefore, 
TABLE VI. Binding energy per atom $\left(E_{b}\right)$ of $\mathrm{Cr}_{n}$ clusters, energy gain $\Delta E$ in adding a $\mathrm{N}$ atom to a $\mathrm{Cr}_{n}$ cluster, and the total magnetic moments of $\mathrm{Cr}_{n}$ and $\mathrm{Cr}_{n} \mathrm{~N}(n \leqslant 5)$. The energies and magnetic moments are given in $\mathrm{eV}$ and $\mu_{\mathrm{B}}$, respectively.

\begin{tabular}{cccccc}
\hline \hline & \multicolumn{2}{c}{$\mathrm{Cr}_{n}$} & & \multicolumn{2}{c}{$\mathrm{Cr}_{n} \mathrm{~N}$} \\
\cline { 2 - 3 } \cline { 5 - 6 } $\mathrm{N}$ & $E_{b}(\mathrm{eV})$ & $\mu_{\text {total }}\left(\mu_{\mathrm{B}}\right)$ & & $\Delta E(\mathrm{eV})$ & $\mu_{\text {total }}\left(\mu_{\mathrm{B}}\right)$ \\
\hline 1 & - & 6 & & 5.15 & 3 \\
2 & 0.97 & 0 & & 5.33 & 9 \\
3 & 0.98 & 6 & & 6.78 & 13 \\
4 & 1.34 & 0 & & 6.76 & 9 \\
5 & 1.50 & 2 & & 7.21 & 3 \\
\hline \hline
\end{tabular}

large and the total moment is reduced to only $3 \mu_{\mathrm{B}}$. This is not too different from the $2 \mu_{\mathrm{B}}$ magnetic moment of the bare $\mathrm{Cr}_{5}$ cluster.

These results point to some common features: (1) $\mathrm{N}$ is bonded to only three $\mathrm{Cr}$ atoms. (2) The coupling of $\mathrm{N}$ to the nearest-neighbor $\mathrm{Cr}$ is antiferromagnetic. Hence all $\mathrm{Cr}$ atoms nearest neighbor to $\mathrm{N}$ are coupled ferromagnetically. (3) Consequently, $\mathrm{Cr}_{3} \mathrm{~N}$ has the largest magnetic moment of all the clusters studied. This result is different from those of the $\mathrm{Mn}_{n} \mathrm{~N}$ cluster where much larger magnetic moments were found. ${ }^{5}$

Our result may have some significance for the understanding of ferromagnetism in Cr-doped GaN. Since the bonding of $\mathrm{Cr}$ to $\mathrm{N}$ is strong, it is expected that in $\mathrm{GaN}$ crystals the doped $\mathrm{Cr}$ atoms may cluster around N. Since the concentration of $\mathrm{Cr}$ in $\mathrm{GaN}$ is small (typically less than $10 \%$ ), it is also expected that the size of the $\mathrm{Cr}_{n} \mathrm{~N}$ clusters in $\mathrm{GaN}$ cannot be large. Since $\mathrm{Cr}$ atoms are antiferromagnetically coupled to $\mathrm{N}$ and $\mathrm{N}$ can have only three nearestneighbor $\mathrm{Cr}$ atoms, the magnetic moment of $\mathrm{Cr}_{3} \mathrm{~N}$ is the largest in the series. Thus we expect that small clusters of $\mathrm{Cr}$ around $\mathrm{N}$ with giant magnetic moments could give rise to the onset of ferromagnetism with a large Curie temperature. Calculation of the Curie temperature of $\mathrm{Cr}$-doped $\mathrm{GaN}$ based upon this clustering idea would certainly be very helpful.

\section{SUMMARY}

The equilibrium geometries, electronic structure, and magnetic moments of $\mathrm{Cr}_{n}$ and $\mathrm{Cr}_{n} \mathrm{~N}(n \leqslant 5)$ clusters in their ground states as well as for low-lying isomers have been calculated using the DFT-GGA method. The geometries were optimized for different spin multiplicities without symmetry constraints. Our results can be summarized as follows: (1) $\mathrm{Cr}_{n}$ clusters are antiferromagnetically coupled with total magnetic moments of $0 \mu_{\mathrm{B}}, 6 \mu_{\mathrm{B}}, 0 \mu_{\mathrm{B}}$, and $2 \mu_{\mathrm{B}}$ for $n$ $=2,3,4$, and 5 , respectively. (2) We found significant differences between the ground-state structures of $\mathrm{Cr}$ clusters with those reported by Chen and Wang. ${ }^{7}$ For example, no dimerlike growth mode was found for clusters containing more than four atoms. It was shown earlier ${ }^{13}$ that the $\mathrm{Cr}_{8}$ cluster also does not exhibit a dimer growth pattern. (3) The structures of $\mathrm{Cr}_{n}$ clusters are substantially modified when doped with a nitrogen atom. The $\mathrm{N}$ atom binds to three $\mathrm{Cr}$ atoms in keeping with its trivalent character. (4) The doping of nitrogen also drastically modifies the magnetic properties of $\mathrm{Cr}_{n}$ clusters. The nearest-neighbor $\mathrm{Cr}$ atoms are coupled antiferromagnetically to the $\mathrm{N}$ atom and hence ferromagnetically with each other. Thus all $\mathrm{Cr}$ atoms in $\mathrm{Cr}_{2} \mathrm{~N}$ and $\mathrm{Cr}_{3} \mathrm{~N}$ are ferromagnetically coupled while without $\mathrm{N}$ the coupling is antiferromagnetic. This results in giant magnetic moments of small $\mathrm{Cr}_{n} \mathrm{~N}$ clusters. For example, the magnetic moments of $\mathrm{Cr}_{2} \mathrm{~N}$ and $\mathrm{Cr}_{3} \mathrm{~N}$ are, respectively, $9 \mu_{\mathrm{B}}$ and $13 \mu_{\mathrm{B}}$ while in $\mathrm{Cr}_{2}$ and $\mathrm{Cr}_{3}$ they are $0 \mu_{\mathrm{B}}$ and $6 \mu_{\mathrm{B}}$. As the $\mathrm{Cr}$ content increases, the $\mathrm{Cr}$ atoms not forming nearest neighbors to $\mathrm{N}$ no longer are forced to couple ferromagnetically with other $\mathrm{Cr}$ atoms. Thus, in larger $\mathrm{Cr}_{n} \mathrm{~N}$ clusters, the total magnetic moments are not strongly influenced by N. (5) The binding of $\mathrm{N}$ and $\mathrm{Cr}$ is substantially larger than that between the $\mathrm{Cr}$ atoms. Thus clustering of $\mathrm{Cr}$ around $\mathrm{N}$ is energetically favorable. This observation may have relevance to studies of $\mathrm{Cr}$-doped $\mathrm{GaN}$, which has been found to be ferromagnetic. In this system, it is possible that $\mathrm{Cr}$ atoms could cluster around $\mathrm{N}$. Since such clusters carry giant magnetic moments, it is possible that Curie temperatures could be enhanced since it is proportional to the square of the moment. We hope that our prediction of $\mathrm{N}$-induced ferromagnetism in very small $\mathrm{Cr}$ clusters will encourage experimentalists to probe the magnetic structure of $\mathrm{Cr}_{n} \mathrm{~N}$ clusters through Stern-Gerlach and/or photodetachment spectroscopy.

\section{ACKNOWLEDGMENT}

This work was supported in part by a grant from the Department of Energy (No. DEFG02-96ER45579).

\footnotetext{
${ }^{1}$ S. M. Casey and D. G. Leopold, J. Phys. Chem. 97, 816 (1993), and references therein.

${ }^{2}$ S. K. Nayak and P. Jena, Chem. Phys. Lett. 289, 473 (1998).

${ }^{3}$ W. Wernsdorfer, N. Alliaga-Alcalde, D. Hendrickson, and G. Christou, Nature (London) 416, 406 (2002).

${ }^{4}$ T. Dietl, H. Ohno, F. Matsukara, J. Cibert, and D. Ferrand, Science 287, 1019 (2000); Y. Ohno, D. K. Yound, B. Beschoten, F. Matsukara, H. Ohno, and D. D. Awschalom, Nature (London) 402, 790 (1999); M. E. Overberg, C. R. Abernathy, and S. J. Pearton, Appl. Phys. Lett. 79, 1312 (2001); M. L. Reed, N. A. El-Masry, H. H. Stadelmaier, M. K. Ritums, M. J. Reed, C. A. Parker, J. C. Roberts, and S. M. Bedair, ibid. 79, 3473 (2001); S. Sonoda, S. Shimizu, T. Sasaki, Y. Yamamoto, and H. Hori, J. Cryst. Growth (to be published).

${ }^{5}$ B. K. Rao and P. Jena, Phys. Rev. Lett. 89, 185504 (2002).

${ }^{6}$ S. E. Park, H. J. Lee, Y. C. Cho, S. Y. Jeong, C. R. Cho, and S. Cho, Appl. Phys. Lett. 80, 4187 (2002).

${ }^{7}$ H. Cheng and L. S. Wang, Phys. Rev. Lett. 77, 51 (1996).

${ }^{8}$ M. J. Frisch et al., computer code GAUSSIAN 98 (Gaussian, Inc., Pittsburgh, 1998).

${ }^{9}$ C. Kohl and G. F. Bertsch, Phys. Rev. B 60, 4205 (1999).

${ }^{10}$ D. Hobbs and J. Hafner, J. Phys.: Condens. Matter 13, L681 (2001).

${ }^{11}$ G. Kresse and D. Joubert, Phys. Rev. B 59, 1758 (1999); D. Hobbs, G. Kresse, and J. Hafner, ibid. 62, 11556 (2000).

${ }^{12}$ M. B. Knickelbein, Phys. Rev. A 67, 013202 (2003).

${ }^{13}$ B. V. Reddy, S. N. Khanna, and P. Jena, Phys. Rev. B 60, 15597 (1999).
} 